\title{
Norois
}

Environnement, aménagement, société

\section{L'action foncière : une ressource pour des mobilisations riveraines}

L'exemple de la requalification patrimoniale d'un lieu de conflits dans la presqu'île de Saint- Tropez

Land policy: a resource for local resident action. The example of the heritage reclassification of a site of conflict on the Saint-Tropez peninsula

\section{Jean-Baptiste Chabert}

\section{OpenEdition Journals}

Édition électronique

URL : https://journals.openedition.org/norois/5855

DOI : $10.4000 /$ norois. 5855

ISBN : 978-2-7535-5227-2

ISSN : $1760-8546$

Éditeur

Presses universitaires de Rennes

Édition imprimée

Date de publication : 17 octobre 2016

Pagination : 43-58

ISBN : 978-2-7535-5222-7

ISSN : 0029-182X

Référence électronique

Jean-Baptiste Chabert, "L'action foncière : une ressource pour des mobilisations riveraines », Norois [En ligne], 238-239 | 2016, mis en ligne le 17 octobre 2018, consulté le 14 janvier 2022. URL : http:// journals.openedition.org/norois/5855; DOI : https://doi.org/10.4000/norois.5855 


Presses
Universitaires
de Rennes
www.pur-editions.fr
Revue en ligne : http://norois.revues.org

\title{
L'action foncière : une ressource pour des mobilisations riveraines L'exemple de la requalification patrimoniale d'un lieu de conflits dans la presqu'île de Saint-Tropez
}

\author{
Land Policy: A Resource for Local Resident Action \\ The Example of the Heritage Reclassification of a Site of Conflict \\ on the Saint-Tropez Peninsula
}

Jean-Baptiste Chabert

Laboratoire CHERPA (Croyances, Histoire, Espaces, Régulation Politique et Administrative), Institut d'Études Politiques d'Aix-en-Provence - 25 rue Gaston-de-Saporta, 13625 Aix-en Provence, cedex 1. (jbchabert@orange.fr)

Résumé : Cet article s’intéresse à un lieu disputé pendant quarante ans entre promoteurs, élus locaux et associations de riverains, le site littoral de Pardigon, situé aux confins de la presqu'île de Saint-Tropez. À partir de l'analyse d'une dizaine d'entretiens auprès de responsables administratifs et associatifs et d'archives associatives, il s'agit de vérifier combien les dispositifs de protection foncière du littoral offrent des ressources à une mobilisation associative de riverains aisés permettant une requalification patrimoniale du lieu. Cette redéfinition s'effectue à la faveur d'une alliance informelle avec des acteurs administratifs marginaux, professionnels du droit et conservateur du littoral, qui leur permet de stabiliser une coalition environnementale.

\begin{abstract}
This article focuses on the coastal site of Pardigon, situated on the borders of the Saint-Tropez peninsula, in south-eastern France. For 40 years, this site has been the subject of disputes between developers, local elected representatives and local residents' associations. Based on the analysis of around 10 interviews with local-authority workers, the leaders of local associations and those responsible for managing the archives of these associations, this article aims to verify the extent to which coastal land-protection policies have provided associations of wealthy local residents with the resources necessary to obtain a reclassification of the site in terms of the heritage status of its land assets. This redefinition of the site is the result of an informal alliance with marginal administrative stakeholders, legal professionals and the coastal protection agency, which has made it possible to establish a stable environmental coalition.
\end{abstract}

Mots clés : politique foncière - Saint-Tropez - littoral - patrimonialisation - riverains - mobilisation - conflits

Keywords: land policy - Saint-Tropez - coast - heritage status - local residents - mobilization - conflict

\section{INTRODUCTION}

Les politiques foncières, c'est-à-dire les activités publiques d'allocation et d'organisation de l'usage du sol, constituent un terrain privilégié d'observation des conflits de lieux. En effet, ce type d'action publique est particulièrement conflictuel, à plus forte raison dans une région caractérisée par une importante spéculation immobilière comme la région Provence-Alpes Côte d'Azur, spécialement dans sa partie littorale. 
Dès le XVIII siècle, les rivages méditerranéens ont été recherchés à des fins touristiques. La douceur du climat, le ciel azur et les paysages de ses côtes ont attiré une classe aisée, souvent aristocratique, originaire d'Europe du Nord. Au cours du XIX ${ }^{\mathrm{e}}$ siècle, cet essor remarquable et quasi-ininterrompu des activités résidentielles et touristiques du littoral méditerranéen accompagne la production d'identités territoriales à la réputation internationale, comme la «Riviera » de La Spezia à Nice ou la « Côte d'Azur » (Boyer, 2009) de Nice à Hyères. Cette entreprise identitaire, d'origine élitaire à la Belle Époque, s'effacera toutefois à la faveur du développement, initié par des Américains aisés, d'un tourisme d'été dans les années 1920, popularisé en 1936 par les congés payés, puis généralisé au cours des années 1950 avec l'avènement d'un tourisme de masse.

Cette trajectoire de développement touristique qui combine entre-soi élitaire et économie touristique pour classes moyennes caractérise particulièrement la presqu'île de Saint-Tropez. Attachée à partir de la fin des années 1950 à la figure de Brigitte Bardot qui achète en 1958 la propriété « La Madrague » sur la route littorale des Canebiers, la presqu'île devient alors un lieu symbolique du gotha de la grande bourgeoisie (Pinçon et Pinçon-Charlot, 2007) qui y bâtit de somptueuses villas. Dans le sillage de la jet set internationale, elle est également fréquentée par de nombreux touristes, logés selon leurs moyens en camping ou en résidences secondaires, tous attirés par la réputation du lieu. Toutefois cette notoriété attise les convoitises : comme sur les corniches voisines des Maures et de l'Estérel, la protection du littoral tropézien qui s'offre au regard des visiteurs n'est pas le résultat d'une politique consensuelle. Elle est essentiellement le produit de luttes locales (Calderaro, 2012) qui s'ancrent dans des mobilisations multiples.

L'objet de cet article est de confronter, à partir d'un conflit d'usage, les registres d'action d'une mobilisation riveraine à l'épreuve des contraintes et ressources de l'action foncière publique et privée. À mi-chemin entre une perspective néo-marxiste s'intéressant aux groupes d'intérêts fonciers et une approche institutionnaliste mettant l'accent sur les variables institutionnelles, l'on adoptera ici une perspective interactionnelle, pour envisager l'action foncière dans ses rapports avec ses bénéficiaires ${ }^{1}$.

1. Ce travail est issu d'une thèse de doctorat en science politique sur l'action foncière en Provence-Alpes Côte d'Azur (Chabert, 2016).
Plus précisément, l'on défend ici l'hypothèse que les dispositifs fonciers de protection du littoral élargissent les ressources et les soutiens d'une mobilisation de riverains aisés, cristallisée autour d'un conflit de lieu, permettant progressivement la requalification patrimoniale de cet espace. L'on parlera ainsi de patrimonialisation dans la mesure où l'action foncière requalifie le lieu « d'espace naturel remarquable » suite à la contestation riveraine. Il s'agit donc d'un objet d'étude privilégié pour analyser les modes de composition d'un bien public à partir d'une pluralité de rapports au lieu.

Le terrain investigué, celui du projet de golf du domaine de Pardigon dans les communes de La Croix-Valmer et de Cavalaire (figure 1), présente l'avantage de constituer un lieu de conflits sur lequel se sont sédimentées près de quarante années de mobilisations associatives, entre 1974 et 2008. Ces mobilisations ont été étudiées à partir d'un matériau issu d'archives associatives et institutionnelles, ainsi que d'une dizaine d'entretiens auprès de responsables administratifs et associatifs menés en $2012^{2}$.

L'on s'attachera d'abord à présenter le contexte du développement d'une économie résidentielle à l'origine d'un processus de spéculation foncière dans lequel s'inscrit le projet de golf opposant promoteurs et riverains. On analysera ensuite le passage du régime d'action judiciaire centré sur une problématique experte à un régime d'action civique plus collectif porté par la coordination associative opposée au projet. Enfin, l'on montrera comment cette action collective va redéfinir les frontières de l'action foncière via l'intermédiation d'acteurs institutionnels marginaux ${ }^{3}$.

\section{QUAND LA SPÉCULATION FONCIÈRE GAGNE DU TERRAIN...}

Le site de Pardigon est aujourd'hui une vaste coupure dans l'urbanisation du littoral de 87 hectares de terrains s'inscrivant dans un ensemble plus vaste

2. Les responsables associatifs interrogés sont ceux de l'Association pour la sauvegarde des sites de La Croix-Valmer et du Comité de sauvegarde de la baie de Cavalaire qui ont pour certains mis leurs archives personnelles à ma disposition. Les responsables administratifs sont des dirigeants et techniciens des services fonciers de la Région, du Conservatoire du littoral, de la SAFER et de l'Établissement Public Foncier PACA.

3. À la lumière de la sociologie des organisations, l'on peut qualifier ces acteurs administratifs de "marginaux sécants » (Crozier et Friedberg, 1977), c'est-à-dire d'acteurs intervenant dans plusieurs systèmes d'action et pouvant de ce fait jouer un rôle souvent décisif de passeurs ou d'intermédiaires entre des logiques d'action différentes, voire contradictoires. 


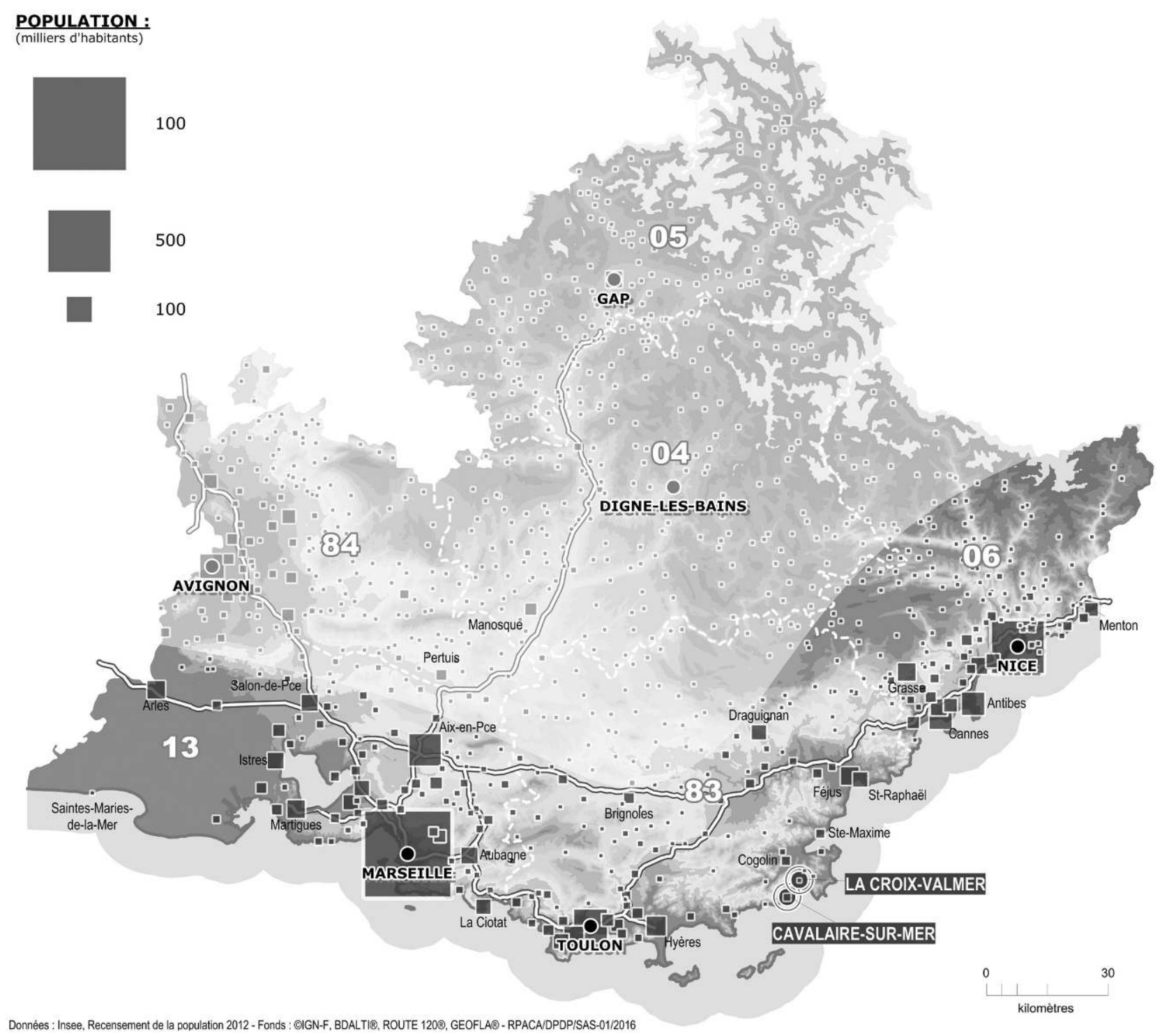

Figure 1 : Localisation de Cavalaire et de La Croix-Valmer sur le littoral azuréen (réalisation : Région PACA, 2016)

Location of Cavalaire and La Croix-Valmer on the French Riviera (produced by Provence-Alpes-Côte d'Azur Regional Council, 2016)

formant, à partir de la ligne de crête, un amphithéâtre qui s'étend du massif des Maures jusqu'à la mer, bordant la baie de Cavalaire (figure 2).

Le nom même du domaine, Pardigon, est attaché à l'histoire locale. Il désigne le propriétaire du terrain au milieu du XIX ${ }^{\mathrm{e}}$ siècle, Alphonse Pardigon, maire, entre 1852 et 1864, de la commune de Gassin à laquelle était alors rattaché le domaine. Il s'agit alors d'une propriété agricole comprenant une ferme construite avant 1808, qui est ensuite complétée au début du $\mathrm{Xx}^{\mathrm{e}}$ siècle d'une forcerie de mimosas et d'une activité viticole ${ }^{4}$. Toutefois, la croissance de la viticulture s'accompagne parallèlement de l'implantation d'activités touristiques de grand standing sur le territoire de la commune, qui devient rapidement une station hivernale et de bains de mer proposant, d'après le guide Joanne de 1906, des maisons à louer

4. Cette activité viticole s'est développée sous l'impulsion de Marcel Ott, ingénieur agronome implanté dans le Var en 1896 qui s'est distingué dans la lutte contre le phylloxéra. L'entreprise Ott est toujours aujourd'hui une des principales exploitations viticoles varoise positionnée dans une production haut de gamme revendiquant cet héritage historique plus que centenaire. 


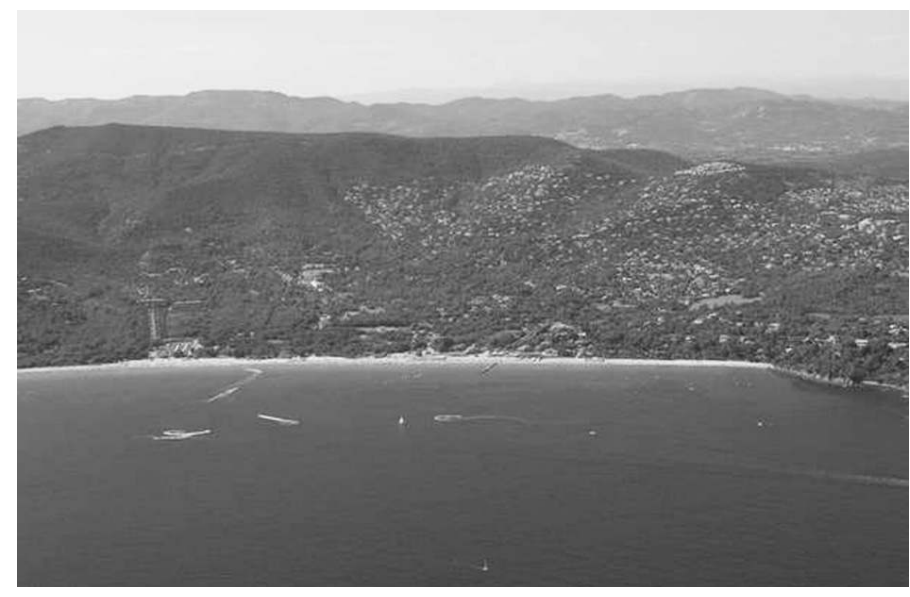

Figure 2 : Vue aérienne du site de Pardigon adossé à la corniche des Maures (source : Conservatoire du Littoral et des rivages lacustres)

Bird's-eye view of Pardigon in the foothills of the Massif des Maures (source: Conservatoire du Littoral et des Rivages Lacustres)

et deux hôtels-pensions ${ }^{5}$. Le développement économique de la commune ${ }^{6}$ entraîne alors un sécessionnisme municipaliste puisqu'en 1929 le quartier de Cavalaire, puis en 1934 le quartier de La CroixValmer, se détachent de la commune de Gassin.

La Seconde Guerre mondiale stoppe provisoirement cet essor et la plage de Pardigon est un des principaux théâtres du débarquement allié en Provence le 15 août 1944 qui voit le parachutage des GI's au milieu des vignes du domaine. Cette activité viticole disparait dès les années cinquante face au « nouveau phylloxéra » (Bromberger et Ravis-Giordani, 1977) que constitue la pression immobilière. La réputation internationale de SaintTropez véhiculée par l’image de Brigitte Bardot et le développement du tourisme de masse favorisent en effet une très forte spéculation immobilière qui entraîne la démolition au début des années soixantedix, du Grand Hôtel de Pardigon, rasé au profit d'une résidence touristique. La propriété adjacente de la ferme, constituée essentiellement de parcelles viticoles (fermes et terrains nus) abandonnées,

5. En 1888, Guy de Maupassant, dans son ouvrage Sur l'eau évoque déjà les premières opérations de spéculations immobilières sur la côte des Maures, notamment à Saint-Aygulf. Dans la baie de Cavalaire, on peut noter, sur le site même de Pardigon adjacent à la ferme, l'implantation de l'hôtel Château-Pardigon, lieu d'un tourisme mondain fréquenté par des personnalités comme Marie Curie ou Aristide Briand, tandis que Sarah Bernhardt occupe à proximité la villa Couadan dominant la baie.

6. Ce rayonnement est relayé par l'industrie naissante du septième art qui met en scène cette réputation touristique exotique. En mars 1940, à la veille de l'Occupation, la plage de Pardigon sert ainsi de cadre à l'adaptation cinématographique par Gaby Morlay et Charles Vanel du roman d'inspiration néo-mauresque de l'académicien Jean Aicard, Le Diamant noir. constitue dès lors une friche agricole très convoitée

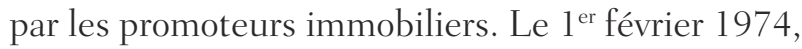
le conseil municipal de La Croix-Valmer approuve ainsi un projet présenté par la société immobilière anglaise Askren Cunningam pour un golf de 18 trous sur 45 hectares et un maximum de 900 logements : c'est le point de départ du conflit.

\section{La formation d'une coalition de croissance de l'économie résidentielle}

Ce programme immobilier bénéficie tout d'abord de l'appui des autorités locales et étatiques. Ainsi, le Conseil municipal de La Croix-Valmer délibère favorablement à plusieurs reprises en faveur du golf, d'abord une première fois, puis une seconde fois en 1975 sur un projet de golf de 18 trous augmenté de $87000 \mathrm{~m}^{2}$ de constructions. Ces volontés locales sont relayées par les services de l'État, alors seuls compétents en matière d'urbanisme et d'aménagement. Le 19 juillet 1976 un arrêté ministériel crée ainsi la Zone d'Aménagement Concerté (ZAC) de Pardigon (figure 3 - planche I).

Cette ZAC, qui s'étend sur une surface globale de 92 ha sur les communes de Cavalaire et de La CroixValmer est confirmée le 30 juin 1980 par un arrêté préfectoral qui approuve le Plan d'Aménagement de Zone pour $87000 \mathrm{~m}^{2}$ de constructions, dont près de $74000 \mathrm{~m}^{2}$ d'habitations, un hôtel de 100 chambres et 50 studios en résidence de tourisme autour de la thalassothérapie. Ces procédures d'aménagement permettent aux autorités publiques de rassembler plusieurs parcelles en friche agricole pour délimiter le foncier et les équipements nécessaires. Elles formalisent l'alliance entre acteurs immobiliers privés et publics, ces derniers souhaitant bénéficier de rentrées fiscales ainsi que d'un renforcement de l'attractivité résidentielle.

Ce projet est en effet soutenu par trois groupes d'acteurs qui influencent le choix des décideurs locaux. Les propriétaires de friches agricoles d'abord, à qui la vente de ces terrains procure une plus-value foncière importante ${ }^{7}$ (Daligaux, 1999). Se constitue ensuite, s'agrège un deuxième groupe autour d'un promoteur immobilier : acteurs du

\footnotetext{
7. Les décennies 1970-1980 sont en effet marquées dans les Maures par une flambée du prix des terrains constructibles creusant l'écart entre le prix des terres agricoles et celui des terrains à bâtir. De nombreuses friches agricoles sont alors requalifiées en terrains à bâtir dans les plans d'occupation des sols (POS)
} 
bâtiment et des travaux publics et professions auxiliaires tels que des architectes, notaires, bureau d'études techniques, géomètres experts et agents immobiliers dont les revenus dépendent directement de la construction. Enfin, le dernier groupe d'acteurs organisé agrège des commerçants, souvent aussi élus locaux, qui apparaissent comme les principaux soutiens au projet, en raison des revenus indirects qu'ils escomptent de ce nouveau complexe résidentiel. L'on propose ainsi de parler de « coalition de croissance ${ }^{8}$ " (Molotch, 1976) de « l'économie résidentielle ${ }^{9}$ " (Davezies, 2008) pour qualifier cette alliance triangulaire entre propriétaires fonciers, acteurs de la promotion immobilière et commerçants qui se forme durablement autour du projet.

À partir de juin 1988, la coalition de croissance est dominée par la société Pierre et Vacances qui, après avoir évincé la société immobilière anglaise, devient le principal opérateur foncier et immobilier de la commune et impose son modèle économique. Le projet de golf change ainsi d'échelle et passe de 900 logements haut de gamme à un aménagement de résidences touristiques d'une capacité totale de 2500 personnes. Ce changement doit se lire comme la consolidation de la coalition de croissance liant les autorités locales et les acteurs privés. Durant cette période, Cavalaire, principale commune d'accueil du projet, est la plus fervente défenderesse du projet à travers son maire Louis Foucher, élu et réélu entre 1982 et 2008 . Cette stabilité politique facilite les négociations locales et permet à Pierre et Vacances de s'imposer durablement. L'expansion de son pouvoir d'influence se traduit par des contacts répétés et directs au plus haut niveau de l'entreprise avec les associations locales et le Conservatoire du littoral qui s'opposent au projet. Cette conduite directe des négociations témoigne de l'hégémonie du groupe dans la maîtrise de l'agenda local. Les

8. Le concept de coalition de croissance est issu des travaux de Harvey Molotch (1976) sur les villes étasuniennes. Cette notion permet de décrire une situation dans laquelle l'orientation des politiques urbaines est déterminée par la structuration des intérêts économiques, notamment fonciers. En effet, selon Harvey Molotch, les propriétaires et les acteurs économiques dont l'activité dépend directement ou indirectement de l'augmentation de la valeur des sols (banques, promoteurs, investisseurs, agences immobilières) ont tendance à chercher à s'allier en vue d'exercer une pression sur les autorités locales afin que celles-ci engagent des projets urbains d'envergure.

9. La notion d'économie résidentielle est empruntée aux travaux de Laurent Davezies (2008) pour caractériser l'économie locale cavalairoise à base touristique, tirant ses ressources de revenus franciliens ou étrangers. cadres dirigeants de la société Pierre et Vacances font ainsi de la baie de Cavalaire un espace d'expansion de leur stratégie. Si les arrangements informels qui président au projet immobilier impliquent les élus locaux, ils n'ont cependant pas de rôle prévalent. C'est bien la coalition de croissance autour du groupe Pierre et Vacances qui joue un rôle dominant dans les options d'aménagement de la baie options que contestent désormais les associations de riverain.

\section{L'ancrage riverain d'une mobilisation associative}

Ce projet de golf cristallise une mobilisation de riverains qui réunit trois associations de propriétaires résidents, rapprochées par le conflit mais aux origines distinctes. D'une part, l'Association pour la sauvegarde des sites de La Croix-Valmer (ASSCV) et le Comité de sauvegarde de la baie de Cavalaire, aux profils initiaux d'associations syndicales de riverains. D'autre part, l'association Vivre dans la Presqu'île de Saint-Tropez, créée plus tardivement en 1986, l'année de l'adoption de la loi Littoral, revendiquant dès le départ ses convictions environnementales ${ }^{10}$. Si ces trois associations de riverains coordonnent leurs efforts judiciaires, elles ne partagent pas la même aire d'influence, comme le signalent leurs appellations. Les liens de proximité sont ainsi plus forts entre le comité cavalairois et l'association de La Croix-Valmer qu'avec l'association tropézienne, dont l'aire d'action dépasse la baie de Cavalaire pour s'étendre à l'ensemble de la presqu'île. Dans cette configuration, l'Association pour la sauvegarde des sites de La Croix-Valmer va occuper une position nodale dans la coordination associative. Cette situation, ainsi que sa conversion environnementale, expliquent notre intérêt pour ses activités militantes.

L'Association pour la sauvegarde des sites de La Croix-Valmer est créée en 1953 et se dénomme alors «Association des propriétaires riverains des plages de La Croix-Valmer ». Comme son nom l'indique alors, il s'agit d'une association créée à l'initiative de propriétaires riverains de plages de La CroixValmer (plages de Mimosa, Gigaro et Héraclée) afin de défendre le caractère familial de la station

10. L'association Vivre dans la presqu'île de Saint-Tropez a ainsi pris une avocate de renom engagée dans la protection de l'environnement en la personne de Corinne Lepage. 
balnéaire ${ }^{11}$. Fonctionnant sous le régime d'une association syndicale libre (ASL), elle reçoit de l'État la concession des plages de Gigaro et d'Héraclée en vue de s'occuper de leur entretien et de leur sécurité $^{12}$. Elle se mobilise alors contre les extractions de sable et de graviers par les entrepreneurs de travaux publics. Elle obtient gain de cause : un gardiennage des plages est instauré en 1958 du ler juillet au 30 septembre (garde municipal bénéficiant d'une gratification de l'association), et des poubelles de plage sont mises en place. D'autres combats sont également menés avec succès contre le développement des sports nautiques (hors-bord et jet-ski), des campings mais aussi des lotissements bordant les plages. L'association appuie alors des revendications particularistes dans la mesure où elle joue le rôle d'un syndic de propriétaires mobilisés sur des conflits d'usages de proximité immédiate avec les plages de Gigaro et d'Héraclée, défendant le bien commun localisé (Lafaye, Thevenot, 1993) d'un groupe restreint n'incluant que des proches voisins, dans une logique d'entre-soi riverain.

\section{DE LA JURIDICISATION DES GRIEFS À L'ENNOBLISSEMENT CIVIQUE : TRAJECTOIRE D'UNE COORDINATION ASSOCIATIVE (1960-2008)}

Ce mode d'action évolue par paliers successifs dans un nouveau contexte d'action publique caractérisé par l'adoption de procédures juridiques de protection du littoral, contribuant à l'ouverture du débat local.

\section{L'apprentissage d'un mode d'action judiciaire (1960-1986)}

Dans un contexte d'urbanisation croissante du littoral, le registre d'action de l'association de la baie de Cavalaire se modifie progressivement à la demande des services de l'État, qui établissent un

11. Une autre dimension familiale est également inscrite dans les statuts de l'association, puisque ceux-ci prévoient une adhésion par famille. D'après François Rémond, le président honoraire de l'association, 400 familles de La Croix-Valmer sont à jour de leur cotisation en septembre 2012. On peut en déduire que près de la moitié de la commune (qui compte environ 3500 habitants) est directement ou indirectement liée à l'association. Le recours à une cotisation familiale permet ainsi d'élargir l'influence de l'association, et témoigne de sa base sociale fondée sur la proximité relationnelle et la logique patrimoniale.

12. Ce n'est qu'à compter des années 1975-1977 que les communes doivent assumer le nettoyage et la surveillance des plages. contact avec une fédération d'associations régionales au profil environnementaliste ${ }^{13}$.

L'évolution de l'ASSCV, d'une association «purement riveraine » vers une association envisageant la nature sous l'angle d'un patrimoine paysager constitutif de l'identité communale, se fait sous l'influence du ministère de l'Équipement, alors seul en charge du littoral. Celui-ci s'intéresse à l'action menée par l'association, consulte ses statuts et suggère leur modification afin de mettre davantage l'accent sur la protection du site. Le régime de l'ASL, qui liait l'adhésion au statut de propriétaires d'un bien riverain, est abandonné au profit de celui d'une association loi $1901^{14}$. Ce lien privilégié entre réseau associatif et services de l'État est caractéristique du champ environnemental où le mouvement associatif a longtemps fait office de service extérieur pour le repérage des situations-problèmes et le suivi des décisions (Lascoumes, 2012). Ce repositionnement en tant qu'association de défense de l'environnement permet en effet à celle-ci de se doter des moyens juridiques nécessaires, notamment en matière d'action judiciaire, dans un contexte de tensions foncières croissantes.

En effet, les premiers grands lotissements de maisons individuelles ont débuté à La Croix-Valmer dès 1955, puis la pression immobilière s'est accentuée avec l'apparition d'opérations groupées, densifiées en constructions et en population comme celle du golf de Pardigon. L'association entre alors en lutte contre des projets récurrents d'urbanisation de la partie occidentale de la presqu'île de SaintTropez fermant la baie de Cavalaire : le cap Lardier. Cette spéculation immobilière, sur un site pourtant classé par le plan directeur du Groupement d'Urbanisme des Maures en «zone forestière protégée » (Daligaux, 1999), suscite l'indignation des membres

13. L'administration centrale encourage la mutation environnementale de l'association car elle souhaite faire aboutir un Plan de Protection de la Côte Varoise, englobant de façon très précise la zone Collebasse/Cap Lardier. L’ASSCV va alors aider la création du Plan d'aménagement de la côte d'Azur qui a, en particulier sauvé certains sites en les classant «zones forestières protégées ». Ce classement est confirmé le 23 avril 1963 par un arrêté ministériel relatif au « Plan directeur du Groupement d’Urbanisme des Maures » qui associe 19 communes du massif et de la côte et procèdera au classement d'une vaste zone autour du cap Lardier en zone touristique et forestière protégée.

14. En 1977, l'association obtiendra l'agrément d'association protectrice de l'environnement prévu par l'article 40 de la loi du 10 juillet 1976 sur la protection de la nature. Il s'agit d'un agrément spécifique pour les associations de protection de l'environnement lui permettant de disposer de facultés particulières pour engager de procédures devant la justice et participer aux débats publics sur les questions d'écologie. 
de l'association. Ces différents dossiers favorisent le développement d'un mode d'action spécifique jusqu'alors peu usité, l'action judiciaire. En 1977, l'association dépose ses deux premiers recours.

L'apprentissage de ce mode d'action s'accompagne de changements dans l'organisation, le recrutement et le cadre argumentatif de l'association. L'élection du bureau de 1972 a ainsi entraîné le renouvellement des 3/4 de ses membres et la modification des statuts, étendant l'intervention de l'ASSCV à toute la commune et supprimant l'article qui liait le nombre de voix à la surface du terrain de la propriété. Cette évolution s'accompagne d'une relative ouverture de l'association que formalise la création d'une catégorie de "membres associés », par opposition à celle de « membres résidents » traduisant le déclin de l'aristocratie foncière. La qualité de propriétaire n'est donc plus le critère déterminant de sélection des adhérents, qui restent toutefois pour la plupart des professions libérales ou des cadres et professions intellectuelles supérieures, ce qui permet à l'association de financer ses activités par les seules cotisations de ses membres. Les principales ressources de l'association demeurent sociales et relationnelles, le carnet d'adresse des membres de l'association permettant de mettre en échec nombre de projets d'aménagement ${ }^{15}$.

Ce relatif élargissement dans le recrutement s'accompagne d'une évolution des activités principales de l'association, puisqu'à partir de 1975 1977 les communes s'occupent de la gestion des plages. Progressivement, l'association quitte son objet syndical de propriétaires pour s'occuper de problèmes environnementaux. Cela se traduit par le développement de nombreux contacts avec les autres organisations de sauvegarde de la nature aux plans départemental et régional, en particulier avec l'Union Régionale pour la protection de la Vie et de la Nature (URVN), créée en 1972, et qui joue le rôle de relais de la Fédération française des sociétés

15. Parmi les différents programmes d'urbanisation projetés on peut citer plusieurs dossiers importants d'urbanisation du Cap Lardier, haut lieu du combat mené par l'association de 1963 à 1981 : projets de 60 résidences de grand standing et d'un port privé en 1963, projet de création d'une route de corniche le long de la presqu'île de Saint-Tropez en 1966; projet de création par le club Méditerranée d'un village de vacances sur la commune de Ramatuelle entre la Bastide Blanche et L'Escalet en 1967, projet de « lotissement du Lardier » comprenant un projet de port avec desserte automobile en 1968, projet de lotissement dit du « Cap Mimosa » en 1977 comprenant l'implantation d'un port, d'un hameau de 80 logements et de 60 villas, démolition de construction édifiée sans permis de construire enfin en 1981. de protection de la nature fondée en $1968^{16}$. Cette fédération d'associations mobilise alors un répertoire de "scandalisation ${ }^{17}$ » contre l'urbanisation du litttoral, dont témoigne l'ouvrage écrit en forme de manifeste par le fondateur de l'URVN, « La Côte d'Azur assassinée » ${ }^{18}$, qui a rencontré un écho important auprès de l'opinion publique.

Cette conception de l'environnement reste cependant limitée. Il s'agit d'abord pour ses membres, généralement d'origine citadine, de préserver la qualité de leur cadre de vie et de son patrimoine naturel. Ainsi, en 1974, à l'annonce du projet de golf de Pardigon entre la mer et la route de Cavalaire, le Président de l'ASSCV fait connaître aux autorités la position très favorable de l'association à propos de la création d'un terrain de golf de 70 ha, complété par un hôtel quatre étoiles de 200 chambres et trois hameaux. L'intégration d'une grandeur écologique au sein d'une grandeur domestique traditionnellement mobilisée par l'association procède donc d'une logique d'arrangement entre cités (Boltanski, Thevenot, 1991) ${ }^{19}$. Elle témoigne du caractère incrémental et progressif du processus d'extension de l'organisation vers des activités de protection environnementale.

\section{La judiciarisation du conflit : re-résidentialisation et écologisation de la cause (1986-1996)}

Cette évolution est favorisée à partir de 1986 par la mise en ouvre de la loi Littoral qui offre un « dispositif de gestion d'intérêts divergents » (Lascoumes, 1995) pour les conflits d'usages littoraux. Ce nouveau cadre législatif est mobilisé par l'association Vivre dans la presqu'île de Saint-Tropez, ce qui entraîne un réalignement écologiste des autres associations

16. Le réseau de l'URVN est un espace transactionnel favorable à l'apprentissage collectif des rôles et normes d'action des militants associatifs. En effet, la fédération de l'URVN regroupe des associations de défense des sites luttant contre des projets d'aménagement sur tout le littoral de la région.

17. Pour reprendre la terminologie des grands principes de légitimation de l'action des formes d'action collective identifiés par Michel Offerlé (1998) : le nombre, l'expertise et la scandalisation.

18. Richard R. et Bartoli C., La Côte d'Azur assassinée, Paris, Roudil, 1971. Cet ouvrage est co-écrit avec Camille Bartoli, un des fondateurs et animateurs de l'Association de défense de l'Environnement et de la qualité de vie de Golfe Juan Vallauris.

19. Dans cet article, on emploie le terme "grandeur » au sens de Luc Boltanski et Laurent Thévenot (1991). Il s'agit par là de faire référence aux contraintes de légitimités auxquels sont soumis les acteurs pour grandir leurs actions. 
désormais positionnées contre le projet de golf et rassemblées dans une coordination associative.

La loi Littoral permet en effet aux associations de mobiliser de nouvelles ressources juridiques pour faire valoir leurs prétentions à la défense du site de Pardigon. Elle change le rapport de force juridique puisqu'elle est de prescription nationale et d'application immédiate, même à l'égard d'opérations en cours qui doivent être mises en compatibilité avec ses obligations. Toutefois, les associations doivent lutter pour avoir accès à ce nouveau levier légal compte tenu des résistances qu'opposent les élus et l'administration préfectorale à son application. Face à cette situation d'incertitude juridique et en l'absence de jurisprudence, deux stratégies d'action sont adoptées par les associations de défense de l'environnement. Échaudé par un précédent échec contentieux ${ }^{20}$, le Comité de Sauvegarde de la Baie de Cavalaire entraîne l'ASSCV dans une stratégie de dialogue avec l'administration et de négociation avec le groupe Pierre et Vacances. Cette stratégie n'est pas suivie par Vivre dans la presqu'île de Saint-Tropez, dirigée par des résidents temporaires de La Croix-Valmer ${ }^{21}$, qui n’hésite pas à engager un recours devant le tribunal administratif. Cette action solitaire est conduite sur les conseils de leur avocate, spécialiste en droit de l'environnement et aux convictions écologistes reconnues, Corinne Lepage ${ }^{22}$. Par son entremise, les membres de cette association disposent donc d'un « capital procédural » (Spire et Weidenfeld, 2011) lui permettant de prendre l'ascendant sur les autres associations mobilisées. Le 23 avril 1992, le tribunal administratif de Nice annule le Plan d'Aménagement de Zone (PAZ) et, par voie de conséquence, les permis de construire accordés par la mairie de La

20. En août 1980, le Comité de sauvegarde de la baie de Cavalaire avait déposé une requête en annulation contre l'arrêté préfectoral approuvant le Plan d'Aménagement de Zone de la ZAC de Pardigon. Ce recours a été rejeté successivement par le Tribunal Administratif de Nice en 1981 puis par le Conseil d'État en 1985.

21. Si des caractéristiques sociales (engagement, réseau, appartenance sociales) prédisposent à ce type de mobilisation, l'on peut souligner que le statut de résident temporaire semble favoriser ici une action plus contestataire contournant les échanges avec les autorités locales que privilégient les dirigeants des associations résidant dans les communes de manière permanente.

22. Corinne Lepage est une figure éminente de la mobilisation environnementale. Le cabinet Huglo-Lepage où elle exerce à partir de 1978 est le premier cabinet d'avocats spécialisé dans le droit de l'environnement. Elle s'est illustrée par son combat contre la société pétrolière Amoco à propos du naufrage de l'Amoco-Cadiz. Cette notoriété lui vaut d'être nommée ministre de l'Environnement sous le gouvernement d'Alain Juppé entre 1995 et 1997.
Croix-Valmer en application dudit PAZ ${ }^{23}$. Les avocats ont donc joué à deux titres un rôle déterminant dans la reconnaissance et la légitimation de la cause environnementale : d'abord en permettant la qualification du projet de golf comme problème public, ensuite en contribuant de manière décisive à la désignation des responsabilités publiques engagées.

Le groupe Pierre et Vacances prépare alors un nouveau projet d'aménagement avec un programme de $45000 \mathrm{~m}^{2}$ de construction, essentiellement fondé sur des résidences de tourisme. Ce nouveau rebondissement suite au précédent succès devant les tribunaux entraîne une convergence des mouvements associatifs autour de la cause environnementale. En janvier 1993, l'ASSCV, le Comité de la Baie de Cavalaire et Vivre dans la Presquî̀le de Saint-Tropez participent à la fondation d'une «Coordination des Associations de Sauvegarde de la Presqu'île de Saint-Tropez ». Ce regroupement associatif favorise le développement d'une argumentation plus générale permettant d'enrôler des acteurs de niveau supra-communal, au-delà des limites du voisinage riverain (Lafaye, Thévenot, 1993). Face aux ambitions renouvelées de Pierre et Vacances, les trois associations forment alors une coordination associative mettant en œuvre une stratégie globale à partir d'une justification écologique commune. La qualification d'« espace naturel remarquable » fournit alors aux associations la ressource juridique nécessaire pour stopper le projet immobilier de Pierre et Vacances. Saisi par une action conjointe des trois associations, le tribunal administratif de Nice déclare ainsi le 4 juillet 1996 le site de Pardigon « espace remarquable » au titre de l'article L 146-6 du Code de l'urbanisme. Bien que frappé d'appel et de cassation, cet arrêt sera par la suite toujours confirmé.

Ce succès contentieux vient donc consacrer un processus d'apprentissage ayant transformé les associations de sauvegarde de l'environnement en « usagers réguliers » (Galanter, 1974) du tribunal administratif. L'analyse diachronique de cette coordination associative rend compte d'un processus de requalification de la cause : la justification domestique qui fondait l'intérêt des riverains s'imbrique progressivement avec une justification écologique sous l'effet de la mobilisation d'un registre d'action judiciaire.

23. Cette décision est confirmée par le Conseil d'État le 10 juillet 1995. 


\section{L'ennoblissement civique des militants associatifs (1996-2008)}

En dépit de cette régulation juridique, la montée en conflictualité se poursuit sur le domaine de Pardigon entre la coalition de croissance et ses opposants qui élargissent leur registre d'action.

Suite à la décision du tribunal administratif de Nice de juin 1996, les deux municipalités publient dans leurs bulletins de juillet un numéro « spécial golf de Pardigon », livrant à la vindicte populaire les responsables des associations. Désignés comme les " deshonoris causa de la baie de Cavalaire », leurs photos sont accompagnées de leurs adresses et de leurs numéros de téléphone mettant en évidence le domicile extra-municipal de certains d'entre eux. Cette stratégie vise à les discréditer en les désignant comme des «fondamentalistes de l'urbanisme et de l'environnement ». Le journal souligne leur origine étrangère au village en vilipendant ces « extrémistes du Nord » qui ont fait construire leur « résidence secondaire sur des emplacements géographiquement remarquables » et qui interdisent « à quiconque de profiter du panorama exceptionnel dont ils ont joui toute leur vie». Insistant fortement sur les justifications économiques du projet qui aurait permis de créer 420 emplois permanents, le journal appelle à une réunion publique sur le sujet.

Cette mise en accusation publique provoque en retour une évolution des registres d'action. Tout en contestant sur le plan pénal les propos diffamatoires, les présidents d'associations décident en effet de jouer le jeu de la justification civique et répondent à l'invitation des deux conseils municipaux de Cavalaire et de La Croix-Valmer le 20 août 1996 réunis en salle des fêtes. Dans cette conjoncture critique, le coup tactique des élus municipaux visant à disqualifier et impressionner les associations locales va paradoxalement se retourner contre eux, le public de la salle soutenant in fine les revendications associatives $^{24}$. Cette réunion constitue le point de départ d'un réajustement du registre d'action associatif autour d'un principe de légitimité citoyenne. De fait, les cadres dirigeants l'ASSCV s'engagent dans la participation à des dispositifs délibératifs et des débats publics. Ainsi, après avoir participé activement aux travaux du SCOT de

24. C'est du moins la version qu'en donne les responsables associatifs : entretien François Rémond et Henri Bonhomme, 7 septembre 2012, p 14.
Saint-Tropez, les cadres dirigeants de l'ASSCV promeuvent désormais le principe de participation et ont contribué à la création d'un collectif associatif sur la commune de La Croix-Valmer, intervenant sur des causes civiques multiples. L'ASSCV s'est par exemple mobilisée sur le problème de la dette de la commune, qui a crû significativement en raison de l'indemnité résultant du contentieux de Pardigon. L'on assiste donc à un processus de recomposition de la cause environnementale autour d'un régime d'engagement civique qui se trouve placé au cœur des controverses locales, notamment lors des élections municipales ${ }^{25}$.

Si cette évolution témoigne des dynamiques du conflit, elle est également rendue possible par la trajectoire sociale des dirigeants associatifs engagés dans ces recompositions. Le cas de François Rémond, Président de l'ASSCV depuis 1992, puis président d'honneur à partir de 1998, est à cet égard très significatif. Il cumule en effet plusieurs types de ressources qui le qualifient particulièrement pour cette entreprise de patrimonialisation du lieu. François Rémond dispose tout d'abord d'une formation juridique et d'une expérience à la Fédération des assureurs, puis dans le secteur bancaire, doublée d'une expérience associative au sein de Conseils syndicaux puis politique au Conseil du $\mathrm{XV}^{\mathrm{e}}$ arrondissement de Paris. Venu s'installer définitivement à Cavalaire à la retraite en 1990, il n'y est toutefois pas inconnu. Membre de l'ASSCV depuis 1960, il peut faire valoir des ressources d'autochtonie par sa belle-famille, propriétaire depuis 1915 du terrain de sa résidence secondaire. Il va dès lors militer activement au sein de l'association contribuant à articuler la contestation autour de Pardigon avec les revendications catégorielles d'un groupe de résidents de classes sociales aisées ${ }^{26}$. Pour la plupart installés depuis plusieurs dizaines d'années dans des maisons individuelles de standing avec jardins et vues

25. Ce réalignement autour d'un régime d'engagement civique se manifeste dans les deux communes lors des élections municipales de 2014, qui sont marquées par des alternances municipales. Le problème de la dette et des augmentations d'impôts résultant du contentieux de Pardigon ont ainsi été au cœur des élections municipales de Cavalaire, tandis que celles de La Croix-Valmer ont été annulées en juin 2014 suite à une requête du précédent maire, ce qui témoigne de la conflictualité de la situation politique de ces municipalités jusqu'alors plutôt stables.

26. Bien que nous n'ayons pas cherché à accéder au fichier des adhérents, les membres de ces associations que nous avons identifiés sont issus des classes sociales supérieures. Assureur, notaire, ingénieur, professeur des universités, héritier de riches familles du capitalisme industriel du nord de la France... sont autant de membres actifs de ces associations. 


\begin{tabular}{|c|c|c|}
\hline Périodes & Coalition de croissance & Coalition anti-croissance \\
\hline 1960-1986 & $\begin{array}{l}1960 \text { : premiers projets immobiliers dans la baie } \\
1974 \text { : approbation du projet de golf de la société Askren } \\
\text { Cunningam par la commune de La Croix Valmer } \\
1976 \text { : arrêté ministériel créant la ZAC de Pardigon } \\
1980 \text { : arrêté préfectoral approuvant le PAZ de la ZAC } \\
\text { Pardigon }\end{array}$ & $\begin{array}{l}1960 \text { : modification du statut de l'ASSCV à la demande } \\
\text { de l'État } \\
1981 \text { : le tribunal administratif de Nice rejette le } \\
\text { recours du Comité de sauvegarde de la baie de Cava- } \\
\text { laire contre le PAZ } \\
1985 \text { : le Conseil d'Etat confirme le rejet du recours }\end{array}$ \\
\hline \multicolumn{3}{|c|}{ Janvier 1986 : vote de la loi Littoral } \\
\hline 1986-1996 & $\begin{array}{l}1988 \text { : la société Pierre et vacances présente un nou- } \\
\text { veau projet de golf } \\
1993 \text { : nouveau projet de résidences touristiques de } \\
\text { Pierre et Vacances }\end{array}$ & $\begin{array}{l}1992 \text { : annulation par le tribunal administratif de Nice du } \\
\text { Plan d'Aménagement de Zone (PAZ) suite au recours de } \\
\text { Vivre dans la Presqu'île de Saint-Tropez } \\
1993 \text { : fondation de la « Coordination des Associations de } \\
\text { Sauvegarde de la Presqu'île de Saint-Tropez ». } \\
1996 \text { : annulation par le tribunal administratif de Nice } \\
\text { du nouveau projet et classement de Pardigon en « espace } \\
\text { naturel sensible» }\end{array}$ \\
\hline \multicolumn{3}{|c|}{ Juillet 1996 : bulletins « spécial golf de Pardigon » et réaction locale de soutien aux associations } \\
\hline $1996-2008$ & $\begin{array}{l}2002 \text { : projet municipal d'implantation d'un collège et } \\
\text { d'un stade sur le site de Pardigon à Cavalaire } \\
2007 \text { : vente des terrains de Pardigon par Pierre et } \\
\text { Vacances }\end{array}$ & $\begin{array}{l}2004 \text { : décision du TA de Nice stoppant les projets } \\
\text { urbanistiques de la commune de Cavalaire } \\
2004 \text { : contacts des associations avec SAFER, EPF et } \\
\text { Conservatoire du Littoral } \\
2008 \text { : acquisition de Pardigon par le Conservatoire du } \\
\text { Littoral }\end{array}$ \\
\hline
\end{tabular}

Tableau 1 : Phases chronologiques du projet et groupes d'acteurs associés Chronological phases of the project and associated groups of stakeholders

sur $\operatorname{mer}^{27}$, les membres de ce groupe social auquel appartient François Rémond sont caractérisés par leurs origines exogènes et citadines et leur souci de défense d'un cadre de vie naturel muséifié. De ce point de vue, les dispositifs de protection issus de la loi Littoral vont leur offrir de nouvelles ressources leur permettant de désingulariser leur cause et d'imbriquer leur engagement domestique avec des registres environnementaux et civiques. La pluralité des attaches de ce dirigeant associatif a donc contribué à la convergence des causes, permettant à son association d'éviter avec succès la clôture identitaire de l'entre soi (Sawicki, 2003) et l'ouvrant à d'autres soutiens, relayés par des professionnels du droit et de la négociation.

\section{LES AUXILIAIRES DE LA MOBILISATION ASSOCIATIVE : LE JUGE ET LE CONSERVATEUR}

De fait, ces associations constituent en quelque sorte les alliés informels d'acteurs marginaux dans

27. Nous nous appuyons ici sur les entretiens réalisés au domicile des leaders associatifs. le champ institutionnel : le juge administratif et le délégué régional du Conservatoire du Littoral. La coordination associative, le juge et le conservateur du littoral convergent ainsi progressivement pour former une coalition anti-croissance ${ }^{28}$. Elle est fondée sur une triangulation informelle des relations qui déborde les rôles institutionnels des acteurs administratifs obligés de braconner avec les règles régissant leur champ d'action afin de bricoler une action foncière informelle qui s'enracine dans la mobilisation collective des associations.

\section{Le rôle d'un « juge administratif militant » dans la juridicisation des griefs}

La juridicisation de la cause est facilitée par les dispositions de la loi Littoral qui permettent au juge d'étendre son pouvoir d'appréciation et de jouer un rôle

28. Certains auteurs ont montré comment face à des coalitions de croissance, la mobilisation de groupes locaux dans les riches banlieues résidentielles américaines a pu entraîner la formation d' « anti-growth coalitions » : voir notamment : Harloe Mickael, «Switching to the slow lane restraining growth in boom town ", Communication International Conference on comparative regional studies, Sendaï Japon 19-25 septembre 1992 ou Deleon Richard, "The urban antiregime progressive politics in San Francisco », Urban Affairs Quarterly, 27 (4), 1992. 
nodal dans l'ajustement d'intérêts contradictoires, se substituant ainsi aux notables du système local.

Toutefois, l'intervention du juge dans les rapports de force locaux l'amène à être perçu comme un acteur engagé et militant, comme le montre l'exemple d'un magistrat du tribunal administratif de Nice. L'un des membres de la juridiction semble en effet avoir joué un rôle prépondérant : le juge Norbert Calderaro. Commissaire du gouvernement, puis vice-président du tribunal administratif de Nice, ce magistrat s'est spécialisé dans le contentieux issu de la loi Littoral en réalisant l'essentiel de sa carrière au sein de la $2^{\mathrm{e}}$ chambre du contentieux de l'urbanisme et de l'environnement du tribunal administratif de Nice. Auteur de trois livres de doctrine et de jurisprudence sur la loi Littoral ${ }^{29}$, il s'est imposé par son action et son œuvre jurisprudentielle comme l'un des experts nationaux sur le sujet. Dans ces ouvrages, il analyse l'ensemble de la jurisprudence afin de clarifier un certain nombre de notions créées par la loi et faisant régulièrement l'objet de controverses. C'est, ainsi, la notion d'espace remarquable au sens de l'article L 146-6 du code de l'Urbanisme ${ }^{30}$ qu'il mobilise pour faire obstacle aux projets de développement du golf de Pardigon, dont il a à instruire le dossier en tant que commissaire du gouvernement lors du jugement du tribunal administratif de Nice sur la ZAC de Pardigon du 4 juillet $1996^{31}$. Ce délibéré intervient suite à une technique peu usitée par la justice administrative, la visite des lieux, qui témoigne de l'implication du juge Calderaro dans ce dossier.

Le rôle déterminant joué par ce magistrat dans la « juridicisation » des conflits d'usage littoraux va lui valoir une certaine défiance. Le numéro spécial des bulletins locaux de Cavalaire et de La Croix-

29. Calderaro N. et Lacrouts J., Le Littoral, Le Moniteur, Paris, 2005, Calderaro N. et Morand-Deviller J., Loi littoral \& loi montagne: Guide de la jurisprudence commentée, Paris, Les éditions EFE, Paris, 2005, Calderaro N., Loi littoral et Loi montagne, 12 ans de jurisprudence commentée, Paris, Les éditions EFE, Paris, 2000.

30. Aux termes de l'article L 146-6 du Code de l'urbanisme, « les documents et décisions relatifs à la vocation des zones ou à l'occupation et à l'utilisation des sols préservent les espaces terrestres et marins, sites et paysages remarquables ou caractéristiques du patrimoine naturel du littoral et les milieux nécessaires au maintien des équilibres biologiques [...] ».

31. Citons ici les conclusions de l'arrêt du tribunal administratif de Nice du 4 juillet 1996 : « Considérant [...] qu’il résulte de tout ce qui précède que le périmètre de la zone d'aménagement concerté de Pardigon doit être regardé comme s'inscrivant dans un paysage remarquable et caractéristique du patrimoine naturel et culturel du littoral varois, au sens de l'article L 146-6 du code de l'uranisme, qui dit être préservé, qu'ainsi l'arrêté [...] de la zone d'aménagement concerté du golf de Pardigon est entachée d'illégalité et doit, par suite, être annulé ».
Valmer de juillet 1996 intitulé «Spécial Golf de Pardigon » critique sévèrement l'action du juge Calderaro, dénoncée comme le «mépris le plus total et le refus le plus pur de la démocratie ». Les autorités locales et préfectorales s'efforceront d'ailleurs de remettre en cause - sans succès - ce jugement en l'attaquant d'abord en appel, puis en cassation. La confirmation des principes jurisprudentiels posés par le juge Calderaro déplace alors le débat sur le terrain de son procès en légitimité instruit au niveau national. Assimilé à un juge militant, sa personnalité concentre les critiques de certains élus qui s'en font l'écho dans un rapport parlementaire sur l'application de la loi Littoral ${ }^{32}$. Cette mise en cause nominative d'un magistrat dans un rapport parlementaire est assez inédite pour être soulignée. Elle illustre bien la critique du « gouvernement des juges » qui condamne la figure du juge militant au nom du principe de neutralité censé guider les magistrats, et de la supériorité de la légitimité démocratique du suffrage universel.

Au total, si la régulation juridique procédurale permet d'arbitrer des intérêts contradictoires au profit des associations environnementales, l'intervention du juge est toutefois perçue comme militante et donc illégitime par l'une des parties. La disqualification de la solution judiciaire aux yeux des autorités locales bloque une issue consensuelle au conflit, faute d'une médiation reconnue par tous.

\section{La médiation informelle du délégué du Conservatoire de l'espace littoral et des rivages lacustres}

C'est à la faveur de ce contexte qu'un délégué régional du Conservatoire de l'espace littoral et des rivages lacustres (ci-après CdL ou Conservatoire) au profil singulier s'impose comme un intermédiaire en capacité de jouer le rôle de médiateur entre les parties $^{33}$ et d'agréger autour de lui une coopération entre opérateurs fonciers publics.

Créé à la suite de la mission Racine et du rapport «Piquard » de la DATAR en 1973, le CdL,

\footnotetext{
32. «Rapport d'information déposé en application de l'article 145 du règlement par la commission des affaires économiques, de l'environnement et du territoire sur l'application de la loi littoral », Jacques Le Guen, 21 juillet 2004. La composition de la mission d'information fait clairement ressortir le poids des élus littoraux. On peut citer parmi eux Jean-Michel Couve, député et conseiller général du canton de La Croix-Valmer.

33. Ce travail de médiation du CdL est également identifié dans les travaux de Kalaora et Konitz (2004).
} 
établissement public placé sous la tutelle du ministère de l'Environnement, possède un patrimoine de plus de 153000 ha en 2012. Son action repose sur le principe de l'intervention publique sur les marchés fonciers - via l'achat de terrains - à l'aide de prérogatives de puissance publique (préemption et expropriation), afin de mettre à l'abri de l'urbanisation une partie des espaces naturels du littoral. Si l'intervention foncière paraît de prime abord antinomique avec les principes d'une gouvernance fondée sur la contractualisation public/privé et la négociation, le Conservatoire est en pratique un exemple d'action foncière pluraliste, donnant une place importante aux élus locaux dans les organes décisionnels et privilégiant dans ses délégations régionales des alliances territorialisées aux contours multiples (Gérard, 2009) ${ }^{34}$.

Ainsi, sur le pourtour méditerranéen, où en 2006 le CdL possède 63 \% de son patrimoine ${ }^{35}$, la faible division parcellaire a permis des négociations directes avec de grands propriétaires comme à Pardigon. Le Conservatoire s'appuie également sur d'autres acteurs locaux en fonction des configurations locales. Sur la presqu'île de Saint-Tropez où il intervient depuis 1978 (figure 4 - planche II), il s'est ainsi appuyé principalement sur les viticulteurs dans la commune de Ramatuelle, tandis qu'il a privilégié une alliance informelle avec les associations locales de riverains dans les communes de Cavalaire et à La Croix-Valmer (Gérard, 2013) ${ }^{36}$.

Le délégué régional est donc en situation d'interdépendance avec les propriétaires fonciers, les asso-

34. Comme le rappelle Yann Gérard (2009), les élus locaux siègent au sein des deux principaux conseils du CdL : les Conseils de Rivage (CR) et le Conseil d'administration (CA). Cette présence leur permet de « filtrer » les décisions, y compris les plus engageantes comme l'approbation des périmètres autorisés sur lesquels le CdL peut acheter, chaque acquisition restant théoriquement soumise à l'avis du conseil municipal.

35. À la même date PACA représente $21,8 \%$ de la surface maritime totale acquise par le CdL (source Sicel, citée par Gérard, 2013).

36. Le CdL dispose d'un ancrage local ancien à Cavalaire puisque dès 1977 il y bénéficie de son premier don provenant d'une propriétaire cavaléroise, militante de la conservation de la corniche des Maures, Mireille Foncin. Son engagement dans la conservation de la corniche des Maures s'inscrit dans un héritage patrimonial, celui de son père, Pierre Foncin, géographe vidalien, fondateur en 1908 de la "Société des amis des Maures et de l'Estérel », dont le nom reste attaché à la mémoire de ce lieu. En effet, la création de cette société s'inscrit dans le contexte de construction de nouvelles identités régionales à la fin du XIX et au début du $\mathrm{Xx}^{\mathrm{e}}$ siècle. Il s'agit d'une une entreprise identitaire spécifiquement varoise, l'invention des « Maures et de l'Estérel ». Les élites modernisatrices - académiques et économiques - installées sur le littoral varois à la faveur de l'attractivité résidentielle et économique de la Belle Epoque réinventent alors l'identité locale à partir d'un passé sarrasin mythifié, faisant de cette partie de la Provence une terre d'exotisme sauvage néomauresque mise en récits dans plusieurs ouvrages (voir notamment celui de Foncin P., Les Maures et l'Estérel, Paris, Armand Colin, 1910). ciations et les élus locaux, ce qui explique son rôle de médiateur. Ce profil va lui permettre de s’imposer comme un entrepreneur de médiation, et de structurer une entente entre opérateurs fonciers publics sur le dossier de Pardigon au bénéfice du CdL, qui en devient propriétaire en $2013^{37}$. Si cette posture de neutralité apparente lui est parfois reprochée par les militants associatifs, elle n'empêche pas les contacts entre les associations et le délégué du Conservatoire, qui entretiennent des rapports ambivalents ${ }^{38}$. Le délégué régional insiste sur sa volonté de prise de distance, allant jusqu'à disqualifier leur combat écologiste :

« [D]ans le Var ce sont des associations de résidences secondaires [...] ce ne sont pas des écologistes [...]. Mais en même temps, ce sont eux qui faisaient le sale boulot. [...] ils ont joué un peu le rôle des nationalistes en Corse, ils ne faisaient pas péter des maisons mais ils faisaient péter des procès. [...] moi j'ai toujours tenu une distance [...] ce sont des retraités ils n'ont que ça à foutre. [...] des anciens Préfets, des cadres, des chefs d'entreprises [...] on les voyait plus à Paris que [dans le Var] je ne pouvais pas cautionner, mais en même temps ça nous était bien utile, voilà, c'étaient eux qui montaient au créneau ${ }^{39}$. »

La comparaison avec les nationalistes corses est éloquente. Les associations de défense du littoral apparaissent comme les interlocuteurs clandestins du Conservatoire : disqualifiés dans l'espace public mais indispensables à son intervention foncière. Toutefois, si les rapports personnalisés avec les associations sont dévalorisés, ils n'en demeurent pas moins une ressource déterminante. Comme Christian Desplats le reconnaît, il s'appuie sur ces militants associatifs afin de disposer de précieux relais préparant le terrain de son action foncière. Ainsi, dans le cas de Pardigon, le conservateur les a incités à produire leur propre contre-expertise en mettant son carnet d'adresse professionnel à disposi-

37. L'acquisition de ce domaine par la puissance publique se fait suite à la vente de la propriété par Pierre et Vacances en 2007. Elle nécessite un montage partenarial complexe associant successivement, la préemption de la SAFER en 2007, puis le portage foncier de l'Établissement public foncier PACA de 2008 à 2012, avant l'acquisition in fine du domaine de Pardigon par le Conservatoire du littoral en janvier 2013.

38. Pour une description plus détaillée de ces rapports, voir : Desplats C., Batailles en bord de mer, Les défis de la protection du littoral, Document, Yenooa, 2015.

39. Entretien Christian Desplats, 17 juillet 2012, p 19-20. 
tion des militants ${ }^{40}$. La mobilisation d'un répertoire expert s'inscrit donc dans une stratégie de légitimation réciproque dessinant, au-delà du discours institutionnel, les contours d'un échange informel entre militants associatifs et Conservatoire du littoral. Les associations de défense du littoral apparaissent donc comme les auxiliaires informels d'une politique foncière littorale fondée sur l'innovation, bricolant ses interventions au gré des configurations, en s'appuyant sur les groupes bénéficiaires de son action.

Au total, le conservateur s'impose comme un acteur nodal au centre de multiples transactions associant les décideurs, les propriétaires et les associations. Il joue ainsi un rôle d'intermédiaire déterminant dans le montage partenarial associant, successivement, la préemption de la SAFER en 2007, le portage foncier par l'Établissement public foncier PACA de 2008 à 2012, l'acquisition in fine du domaine de Pardigon par le Conservatoire du littoral en janvier $2013^{41}$. Par ailleurs, le rôle déterminant joué par le délégué du conservatoire dans le dossier de Pardigon s'inscrit dans une trajectoire qui consacre Christian Desplats comme un «militant expert » (Nonjon, 2005) contribuant activement à la professionnalisation de la cause du «foncier écologique » et à sa politisation ${ }^{42}$. L'accumulation de ressources relationnelles et d'une expertise écologique suscite alors l'intérêt du milieu partisan écologiste pour les élections régionales de 2010. Son profil hybride plaide ainsi en faveur de son éligibilité et lui permet d'accéder à l'investiture, puis à l'élection sur la liste EELV en l'absence de militantisme partisan antérieur au sein du mouvement écologiste.

40. Par exemple, les associations ont missionné, sur recommandation de Christian Desplats, un bureau d'étude afin qu'il réalise une proposition d'aménagement du site en adéquation avec les principes d'action du CdL : entretien François Rémond et Henri Bonhomme, 7 septembre 2012, p 18. L'expertise est donc une ressource mobilisée ici dans une stratégie de légitimation réciproque.

41. Cette acquisition par le CdL est annoncée en 2008 par Jean-Louis Borloo alors ministre de l'Écologie, du Développement et de l'Aménagement durables qui s'est rendu sur place le 27 février 2008 en compagnie du Président du CdL pour en faire l'annonce officielle ce qui témoigne de l'importance symbolique de l'opération.

42. La trajectoire de Christian Desplat est caractérisée par un processus de professionnalisation militante renversant le modèle de carrière militante suivant lequel l'engagement devient une profession. Au contraire, la profession est pour lui un engagement qui permet d'accéder à une position de «militant-expert » qui le qualifie pour son éligibilité.

\section{Conclusion}

Au terme de cet article, l'on a montré qu'il existe un continuum entre action collective locale et action foncière. Face aux projets immobiliers, les dispositifs de protection du littoral offrent en effet un cadrage normatif et expert aux mobilisations associatives permettant la formation d'une coalition anti-croissance liant informellement une coordination associative, un juge et un conservateur. Cette mise en mouvement des rôles et des postions s'est opérée grâce au travail de deux figures d'intermédiation de la politique foncière: le travail généraliste de traduction juridique du magistrat qui a permis de juridiciser les griefs et le travail de courtage du conservateur qui a permis de négocier l'acquisition par le Conservatoire. Ce conflit autour de la destinée du lieu précipite ainsi l'émergence d'un régime d'action foncière environnementale, qui renforce en retour l'emprise résidentielle des riverains, en même temps qu'il contribue à la politisation écologiste du problème foncier.

\section{Bibliographie}

Banos V., 2009. Réflexion autour de la dimension spatiale des processus normatifs. Exemple de la cohabitation entre agriculteurs et autres usagers en Dordogne, Géographie et cultures, $\mathrm{n}^{\circ} 72$, p. 80-98.

Boltanski L., Thévenot L., 1991. De la justification. Les économies de la grandeur, Paris, Gallimard, 496 p.

Boyer M., 2009. L'hiver dans le Midi, Paris, L'Harmattan, 439 p.

Bromberger C., Ravis-Giordani G., 1977. Le deuxième phylloxéra? Facteurs, modalités et conséquences des migrations de loisir dans la région Provence-Alpes-Côte d'Azur, Marseille, Service Régional de l'Équipement, 216 p.

Calderaro N., 2010. La loi Littoral et le juge, Méditerranée [En ligne], 115, mis en ligne le 30 décembre 2012, consulté le 2 janvier 2014. [http://mediterranee.revues.org/5149].

Chabert J.-B., 2016, Construire la politique foncière en Provence-Alpes-Côte d'Azur (1974-2014). Analyse des interactions au sein d'une action publique, thèse de doctorat de science politique, Aix-Marseille Université-Institut d'Études Politiques d'Aix-en-Provence, 713 p.

Crozier M., Friedberg E., 1977. L'acteur et le système, Les contraintes de l'action collective, Paris, Seuil, 493 p.

Daligaux J., 1999. Urbanisation et société locale en Provence, Paris, L'Harmattan, 241 p.

Davezies L., 2008. La République et ses territoires, Paris, Seuil, $110 \mathrm{p}$.

Deleon R, 1992. The urban antiregime progressive politics in San Francisco, Urban Affairs Quarterly, 27 (4). 
Gaudin J.-P., 2007. Gouverner par contrat, Paris, Presses de Science Po, 275 p.

Galanter M., 1974. Why the "haves" come out ahead : speculation on the limits of legal changes, Law and society review, 9, p. 95-160.

GÉRARD Y., 2009. Une gouvernance environnementale selon l'état? Le conservatoire du littoral entre intérêt général et principe de proximité, in Vertigo - la revue électronique en sciences de l'environnement [En ligne], Volume 9, $\mathrm{n}^{\circ}$ 1, p. 5.

GÉrARd Y., 2013. Intervention foncière publique et protection des espaces naturels. Le Conservatoire du littoral en France méditerranéenne, in Perrin C. (dir.), Un littoral sans nature? L'avenir de la Méditerranée face à l'urbanisation, Rome, Collection de l'École française de Rome, p. 229-242.

Harloe M., 1992. Switching to the slow lane restraining growth in boom town, Communication International Conference on comparative regional studies, Sendaï Japon 19-25 septembre.

Kalaora B., Konitz A., 2004. Le Conservatoire du littoral : entre patrimonialisation et médiation, Annales de Géographie, t. 113, no 635, p. 87-99.

Lafaye C., Thévenot L., 1993. Une justification écologique? Conflits dans l'aménagement de la nature, Revue française de sociologie, 34-4, p. 495-524.

Lascoumes P., 2012. Action publique et environnement, Paris, PUF, 84 p.
Lascoumes P., 1995. Les arbitrages publics des intérêts légitimes en matière d'environnement, Revue française de science politique, vol. 45, n 3, p. 407.

Molotch H., 1976. The City as a Growth Machine: Toward a Political Economy of Place, American Journal of Sociology, vol. $82, \mathrm{n}^{\circ} 2$.

Nonjon M., 2005. Professionnels de la participation : savoir gérer son image militante, Politix, 2005/2 (nº 70), p. 89-112.

Offerlé M., 1998. Sociologie des groupes d'intérêt, Paris, Montchrestien, $158 \mathrm{p}$.

Pinçon M., Pinçon-Charlot M., 2007. Les ghettos du gotha. Comment la bourgeoisie défend ses espaces, Paris, Seuil, 295 p.

Sabatier P., Schlager E., 2000. Les approches cognitives des politiques publiques : perspectives américaines, Revue française de science politique, 50 (2), p. 209-234.

Sawicki F., 2003. Les temps de l'engagement. À propos de l'institutionnalisation d'une association de défense de l'environnement, in Lagroye J. (dir.), La politisation, Paris, Belin, p. 123-146.

Spire A., Weidenfeld K., 2011 , Le tribunal administratif : une affaire d'initiés? Les inégalités d'accès à la justice et la distribution du capital procédural, Droit et société, 2011/3 $n^{0} 79$, p. 689-713. 
planche I (Jean-Baptiste CHABERT - L'action foncière : une ressource pour des mobilisations riveraines)

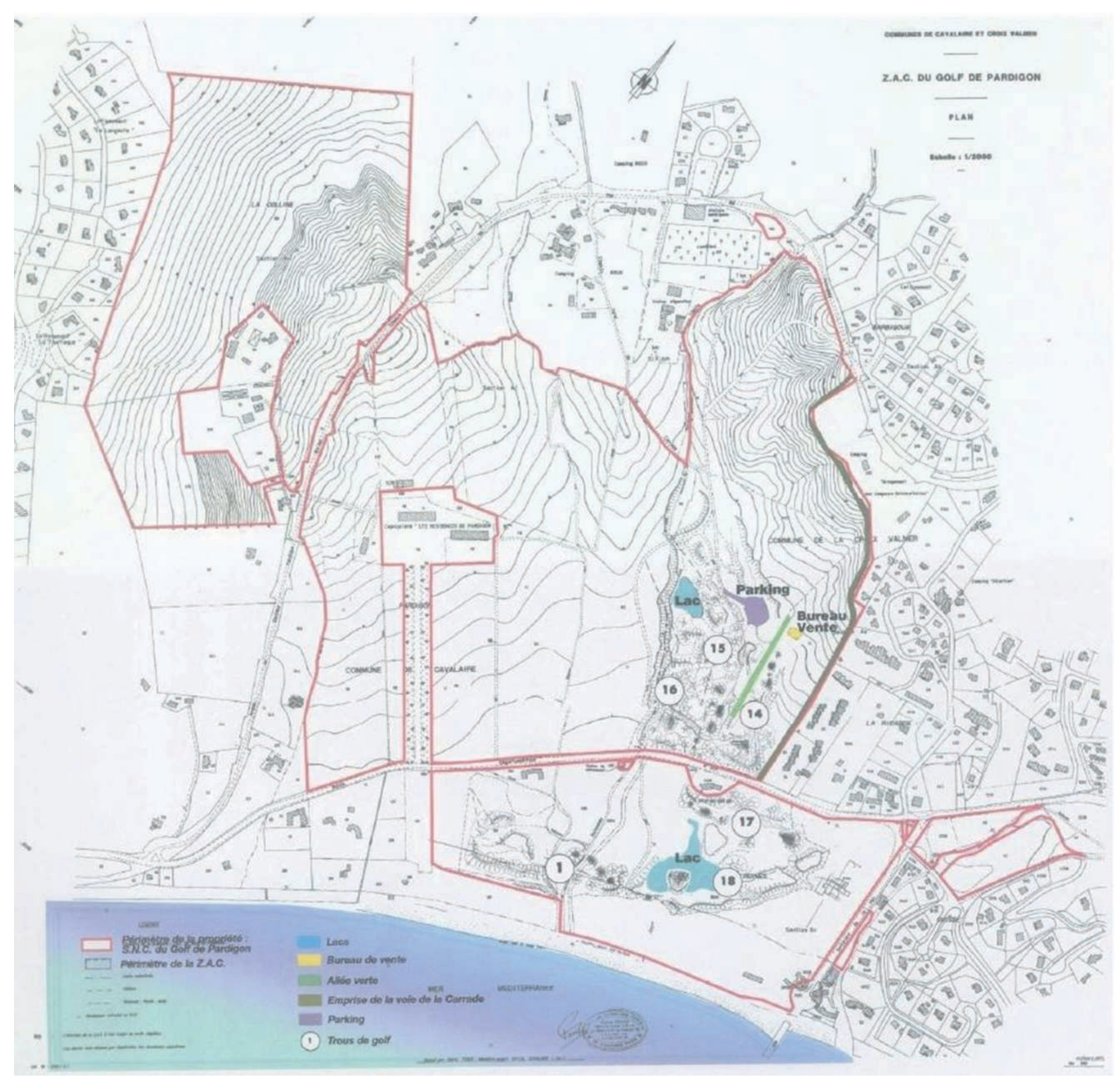

Figure 3 : Plan de la ZAC de Pardigon (source : site du Comité de Sauvegarde de la Baie de Cavalaire)

Map of the Pardigon mixed-development zone ("zone d'aménagement concerté") (source: website of the Committee pour la Sauvegarde de la Baie de Cavalaire) 




Figure 4 : L'action du Conservatoire du Littoral dans la presqu'île de Saint-Tropez (réalisation: Région PACA, 2016) Action undertaken by the Conservatoire du Littoral (French coastal protection agency) on the Saint-Tropez peninsula (produced by Provence-Alpes-Côte d'Azur Regional Council, 2016) 